\title{
Deutliche Realeinkommensverluste für Arbeitnehmer: Die neue Dimension eines Aufschwungs
}

Deutschlands Wirtschaft befindet sich seit 2005 in einem Konjunkturaufschwung, der bis in das Jahr 2008 hineinreicht. Das preisbereinigte Bruttoinlandsprodukt ist bis zum Frühjahr 2008 um rund $9 \%$ gestiegen, die Beschäftigung nahm um fast 3,5 \% zu und die Zahl der Arbeitslosen ging um über 1 Mio. Personen zurück. Dies ist eine Entwicklung, die in den ersten elf Quartalen mit dem vorherigen Aufschwung zwischen 1998 und 2001 vergleichbar ist. Der derzeitige Aufschwung hält jedoch insgesamt länger an. Dennoch: Nach Presseberichten sind die meisten Menschen davon überzeugt, nicht an dem Aufschwung teilzuhaben. Warum ist das so? Hat sich die Lage der Mehrheit der Bevölkerung in dieser Zeit tatsächlich nicht verbessert? Wer hat dann aber profitiert? Eine Analyse des Aufschwungs liefert erste Antworten.

\section{Arbeitsmarkt im Wandel: Die gesellschaftlich- wirtschaftlichen Ursachen des Lohndrucks}

Ausgangspunkt für die Analyse der Realeinkommensentwicklung der vergangenen Jahre ist der deutsche Lohnfindungsprozess. Die Tarifbindung nimmt seit Jahren stetig ab, und innerhalb der Tarifverträge gibt es einen wachsenden Trend zur Flexibilisierung. ${ }^{1}$ Dafür können unterschiedliche Gründe angeführt werden:

(1) Ein wichtiger Faktor ist sicherlich der strukturelle wirtschaftliche Wandel, der von einer Industriegesellschaft mit einem hohen gewerkschaftlichen Organisationsgrad hin zu einer nur noch lose organisierten Dienstleistungsgesellschaft führt. In der Tat bestehen Dienstleistungsbranchen aus kleineren Betrieben, bieten mehr Teilzeitarbeitsplätze, beschäftigen auch mehr Frauen und jüngere Menschen als die industriellen Branchen. Dies alles sind Beschäftigtengruppen, für die bislang eine schwächere Tarifbindung und niedrige Stundenlöhne charakteristisch sind. Wie die Daten von Eurostat zeigen, wird der Unterschied in der Entlohnung zwischen dem industriellen Sektor und dem Dienstleistungsbereich in Deutschland immer größer (Horn et al. 2007). Minijobs, für die sehr niedrige Stundenlöhne bezahlt werden, konzentrieren sich vor allem in den Dienstleistungsbranchen. Bereinigt man die Lohnsteigerungen um den Einfluss von Strukturänderungen, hält man also eine bestimmte Beschäftigungsstruktur eines Stichjahrs (z. B. 1991) in den Folgejahren konstant, dann zeigt sich fast das gleiche gesamtwirtschaftliche Lohnmuster. Die Beschäftigungsverlagerungen vom industriellen Sektor hin zu den Dienstleistungen schlagen allerdings nur mit $3 \%$ bis $5 \%$ kumuliert nach 18 Jahren, also nicht dominant, auf die aggregierten Lohnsteigerungen durch.

(2) Ein anderer Faktor, der direkt am Wirtschaftswandel anknüpft, ist die zunehmende Globalisierung. Die Zerlegung der Produktionsprozesse nimmt zwar verschiedene Formen an, hat letztlich aber dieselbe Konsequenz: Durch die Auslagerung verschiedener Tätigkeiten (IT, Kundenbetreuung, Verwaltungstätigkeiten) außerhalb der Mutterkonzerne werden einem Teil der bisherigen Stammbelegschaft die oft günstigeren Haustarifverträge entzogen. Anschließend haben diese Beschäftigten entweder keinen oder ungünstigere Tarifverträge in externen Firmen (dasselbe betrifft die Mitbestimmungsorgane). Tatsächlich durchgeführte oder auch nur angedrohte Auslagerungen von Arbeitsplätzen ins Ausland führen dazu, dass die Haustarifverträge flexibilisiert werden und/oder die Tariflohnentwicklung gedämpft wird.

(3) Und schließlich spielen die Privatisierung und Deregulierung der öffentlichen Monopole (Post (Brandt et al. 2007), Telekom und Energie) ebenso eine Rolle wie die abnehmende Unterstützung der politischen und ökonomischen Eliten für Ge- werkschaften und Tarifverträge, die sich in Deutschland zeigt (Bispinck 2003). Diese Funktionseliten sahen in erster Linie die Arbeitskosten als Hauptverursacher der Massenarbeitslosigkeit an, was zu einem immer einseitigeren Reformeifer Richtung Deregulierung des Arbeitsmarktes führte und den Weg zu betrieblichen Verhandlungen ebnen sollte (Bispinck/Schulten 2005).

\section{Bisherige Reformen im Überblick}

Wesentliche Arbeitsmarktreformen wurden bereits in den 1990er Jahren implementiert, nachdem sie - gemäß dem

\footnotetext{
1 Dies kann man auf verschiedene Aspekte beziehen: auf die zum Teil sehr niedrigen tariflichen Stundenlöhne (Bispinck 2007), auf die Erhöhung der geregelten Arbeitszeit, die Ausweitung der Arbeitszeitkonten (Bispinck 2005) und die Zunahme der Öffnungsklauseln (Bispinck 1999).
}

Camille Logeay, Dr., Wissenschaftlerin im Institut für Makroökonomie und Konjunkturforschung (IMK) in der Hans-Böckler-Stiftung. Arbeitsschwerpunkte: Makroökonomische Grundlagenforschung und Arbeitsmarkt. e-mail: camille-logeay@boeckler.de Rudolf Zwiener, Dr., Wissenschaftler im IMK in der Hans-Böckler-Stiftung. Arbeitsschwerpunkte: Modellsimulationen und wirtschaftspolitische Beratung.

e-mail: rudolf-zwiener@boeckler.de 
OECD-Institutionenmonitor - schon in den 1980er Jahren auf den Weg gebracht worden waren (Bassanini/Duval 2006). Der Höhepunkt - zumindest wird es in der politischen Debatte so wahrgenommen wurde unter der rot-grünen Regierung mit der Agenda 2010 und den sie begleitenden Hartz-Reformen erreicht (vgl. als Überblick Bothfeld 2007; Ebbinghaus/Eichhorst 2006; Deutsche Bundesbank 2005a, S. 25). Wie sehr die Reformen ab 2001 auch auf die Beschäftigtenstruktur durchgeschlagen sind und sie verändert haben, wird an der Entwicklung der atypischen Beschäftigung besonders deutlich (als Überblick vgl. Keller/Seifert 2007).

Die Reformen tangierten viele der Institutionen des Arbeitsmarktes: Kündigungsschutz, Teilzeit und Zeitarbeit, befristete Beschäftigung. Einige atypische ${ }^{2}$ Beschäftigungsformen wurden arbeitsmarktpolitisch gezielt gefördert: Minijobs, Midijobs, Zeitarbeit (PSA), Ich-AGs, sogenannte Ein-Euro-Jobs (Arbeitsgelegenheiten in der Mehraufwandsvariante). Die Umstrukturierung der Arbeitslosenunterstützung hat mit dem Job-AQTIV-Gesetz (2002) begonnen und ist mit den HartzGesetzen (insb. Hartz IV 2005) vollzogen worden. Die hauptsächliche Zielsetzung hierbei war, die Höhe der Unterstützung zu reduzieren, um Arbeitsanreize zu geben, was - so die Intention des Gesetzgebers auch eine Budgetentlastung (insbesondere der Bundesagentur für Arbeit (BA)) bewirken sollte. Es erfolgte eine Zusammenlegung der Arbeitslosen- und Sozialhilfe. Ferner wurden die Bezugsbedingungen für das neue ALG II verschärft. Doch auch die Zuverdienstmöglichkeiten zum Leistungsbezug und ihre Anrechnungen auf die Leistungshöhe wurden verändert.

Mit den arbeitsmarktbezogenen „Deregulierungsreformen" widerum verband der deutsche Gesetzgeber die Hoffnung, bislang ungenutzte Beschäftigungspotenziale zu erschließen. Als Hauptursachen für das Problem der (verfestigten) Arbeitslosigkeit wurden der „zu kleine“ Niedriglohnsektor (für Geringqualifizierte) und die vermeintlich „zu geringe“ Flexibilität am Arbeitsmarkt gesehen (SVR 2007, S. 10; OECD 2008, S. 79ff.; EU-Kommission 2007, S. 36ff; Deutsche Bundesbank 2007a, S. 3545). Atypische Beschäftigungsformen sollten das Arbeitsangebot im Niedriglohnsektor, also in den Nischen des Arbeitsmarktes, erhöhen und die vermeintliche Inflexibilität regulärer Jobs umgehen. Die logische Folge - eine Zunahme der Lohnungleichheit bei gleichzeitiger Abnahme der Einkommensungleichheit, da nun mehr Arbeitslose einen Arbeitsplatz finden sollten, - war intendiert. Ob eine solche Strategie eine dauerhafte Stärkung des Wachstums bewirkt, ist ohnehin fraglich. Jedoch setzte man auf zumindest vorübergehende positive Wirkungen (ebd.). Ob diese Erwartungen erfüllt worden sind, wird im Folgenden untersucht.

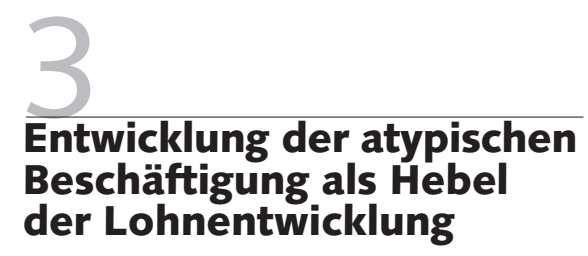

Die Reformen sind - gemessen an den Absichten - insoweit erfolgreich gewesen, als sich die atypische Beschäftigung dynamisch entwickelt hat, insbesondere während der letzten Jahre. So hat sich der Anteil der Zeitarbeit an der sozialversicherungspflichtigen Beschäftigung zwischen 1994 und 2006 vervierfacht $(0,5 \%$ gegenüber 2,2 \% nach Daten der BA) und betraf Mitte 2007 bereits etwa 730.000 Personen. Die Leiharbeitnehmer stehen aber oft schlechter da als die Stammbelegschaft, was ihre Interessenvertretung, die Arbeitsbedingungen und die Lohnhöhe betrifft (Nienhüser/Matiaske 2003; Promberger 2006, 2008).

Befristete Arbeitsplätze sind ebenfalls zahlreicher geworden. Während zwischen 1999 und 2002 noch ein Rückgang zu verzeichnen war auf ein Niveau von $12 \%$, sind im Jahr 2007 bereits 14,6\% der Arbeitsverträge befristet (Eurostat). ${ }^{3}$ Der Anteil an Teilzeitarbeit nimmt ebenfalls stetig zu; der große Anstieg an Minijobs, die per se Teilzeitarbeitsplätze sind, hat natürlich zu diesem Trend deutlich beigetragen. Betrachtet man nur die sozialversicherungspflichtige Beschäftigung (ohne Minijobs und ohne Ein-Euro-Jobs in der Mehraufwandsvariante), dann ist auch dort der Teilzeitanteil von 13,4 \% im Jahr 1999 auf $17,7 \%$ (2007) gestiegen (BA und eigene Schätzung).

Darüber hinaus sind noch weitere Entwicklungen zu nennen, die dazu beigetragen haben, die Löhne in regulärer Beschäftigung unter Druck zu setzen: Die weitreichende Kombilohnregelung durch Miniund Midijobs seit 1999 sowie die Aufstockungsmöglichkeiten von ALG II seit
2005. Die verringerten Arbeitnehmersozialbeiträge, die mit Mini- und Midijobs einhergehen, sind de facto eine Lohnsubvention für Löhne bis $800 €$ pro Monat. Charakteristisch für Minijobs ist, dass sie besonders häufig im Niedriglohnbereich ausgeübt werden bei einem Einkommen, das um Zweidrittel niedriger ist als der Medianstundenlohn (Bosch/Kalina 2007). Deswegen kann angenommen werden, dass die Beitragsreduzierungen letztendlich den Arbeitgebern in Form von niedrigen Bruttolöhnen zugute kamen. Darüber hinaus gibt es Indizien, dass zumindest in den ersten Jahren der 2003er Reform viele sozialversicherungspflichtige Jobs in Minijobs umgewandelt worden sind (Rudolf 2003; SVR 2004; Fertig et al. 2005). Die Frage, ob und inwieweit Minijobs vormals reguläre Beschäftigungsverhältnisse substituiert haben, ist allerdings strittig (verneinend: BA 2004; Deutsche Bundesbank 2005b, S. 40-41). Gleichwohl aber üben diese subventionierten Beschäftigungsformen einen Lohndruck nach unten aus, dessen Sogkraft sich auch in ihrem Wachstum ausdrückt. Die Minijobs haben einen regelrechten Boom nach der zweiten Reform erlebt und zählen inzwischen 4,9 Mio. Personen. Allerdings ist ihre Zahl seit 2005 weniger gestiegen als die der sozialversicherungspflichtig Beschäftigten, was darauf hinweist, dass die Substitutionspotenziale zunehmend ausgeschöpft sind.

Die Möglichkeiten für ALG-II-Bezieher, Grundsicherungsleistungen zusätzlich zum Erwerbseinkommen zu beziehen (Aufstocker), geben den Unternehmen faktisch uneingeschränkte Gelegenheiten, die Löhne auf Kosten der Steuerzahler nach unten zu drücken. Tatsächlich kann ein ALG II beziehender Arbeitloser bis zu 15 Stunden

\footnotetext{
2 Atypisch in verschiedenen Dimensionen: Ihre Abdeckung bei der Sozialversicherung ist niedriger als bei regulärer Beschäftigung, wie es bei den Minijobs, den Ich-AGs oder den Ein-Euro-Jobs der Fall ist (das heißt nicht, dass die Beschäftigten über keine Sozialversicherung verfügen, nur dass die ausgeübte Tätigkeit mit keinen oder geringeren Ansprüchen verbunden ist); Die Kündigungsbedingungen sind leichter (Minijobs, Zeitarbeit) und der Grad der gewerkschaftlichen Organisation ist niedriger (Ich-AG, Mini- und Midijobs, Zeitarbeit).

3 Nimmt man die Abgrenzung und Zahlen von Destatis (2006), die Auszubildende ausschließt, ergeben sich ähnliche Entwicklungen, allerdings auf einem niedrigeren Niveau. So lag die Befristungsquote laut Destatis 2006 bei $10,8 \%$ gegenüber einer Quote von 6,4 \% im Jahr 2002.
} 


\section{Abb. 1: Löhne und Gewinne im Zyklenvergleich}
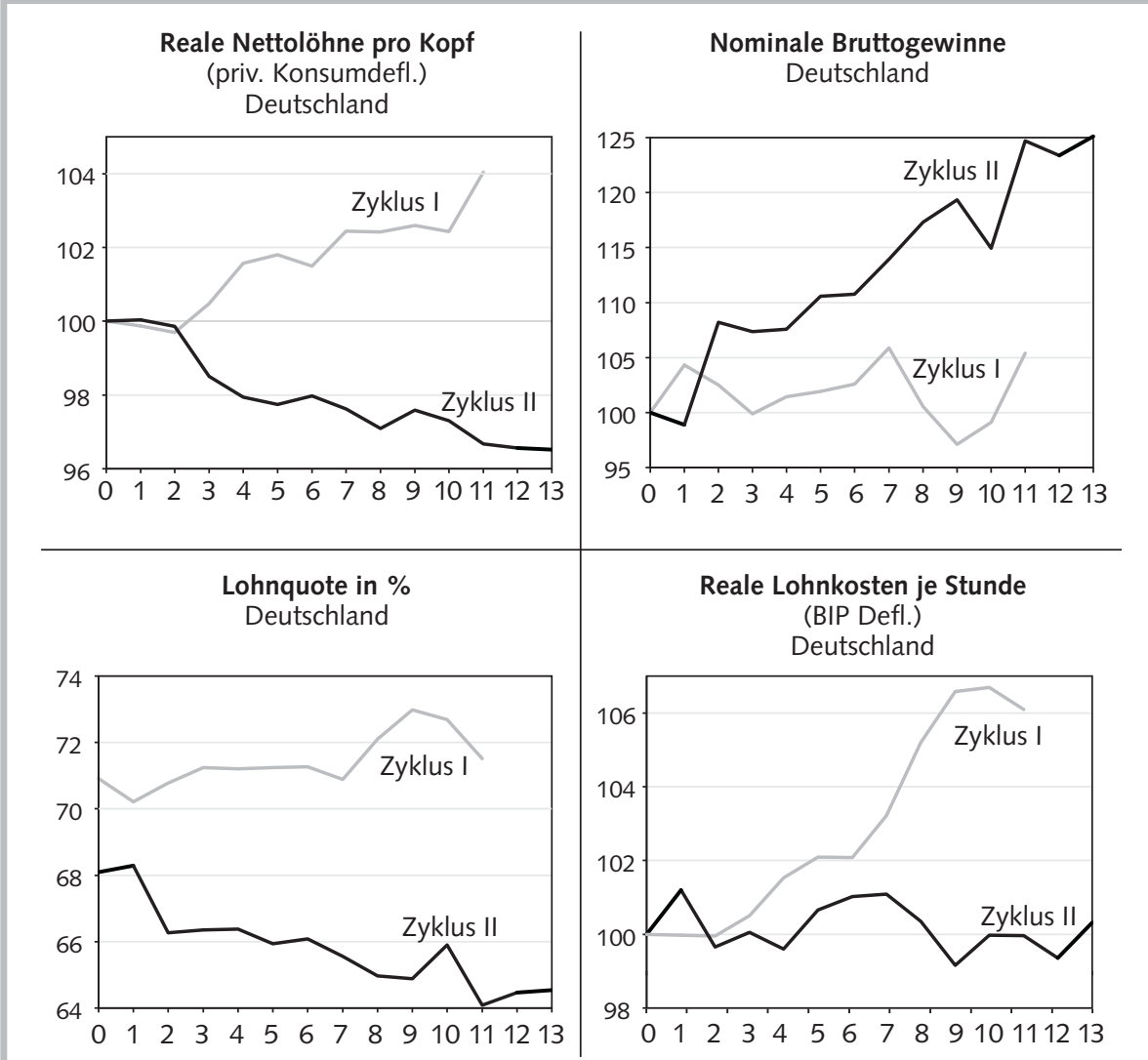

Zyklus I: 1998q2 - 2001q1 (= 12 Quartale), Zyklus II: 2004q4 - 2008q1 (= 14 Quartale). Quelle: Destatis; Berechnungen der Autoren.

pro Woche arbeiten, ohne seinen Arbeitslosenstatus zu verlieren. De facto allerdings sind die Arbeitsstunden ohnehin kein Kriterium stricto sensu, um eine Grundsicherung (ALG II) zu beziehen, die zu einer Form des Kombilohns geworden ist. Wie sich diese Kategorie der Erwerbstätigkeit (Niedriglöhne plus Grundsicherung) weiter entwickelt, ist schwierig einzuschätzen. Aus den Daten der BA kann man allerdings seit 2005 eine steigende Tendenz schlussfolgern: Im Januar 2008 waren bereits 1,2 Mio. ALG-II-Bezieher erwerbstätig. 819.000 von ihnen wurden nicht mehr als Arbeitslose gezählt, weil sie z. B. einer Erwerbstätigkeit von über 15 Stunden pro Woche nachgingen.

Der Lohndruck ist also an verschiedenen Fronten zu spüren: mehr atypische Beschäftigung und mehr Kombilohn. Beides zeigt eine tief greifende Veränderung des deutschen Arbeitsmarktes. Mit dem Lohndruck nach unten öffnet sich auch die Lohnschere immer weiter. Dass Löhne und Einkommen auseinanderdriften, ist für die letzten Jahre ausreichend belegt worden (Brenke 2007; Gernandt/Pfeiffer 2007;
Grabka/Frick 2008; SVR 2007, Tabellen 59-60; Destatis 2006, Kap. II-18; EU-Kommission 2004, S. 168; Rhein/Stamm 2006; Bosch/Kalina 2007). Die neuesten Zahlen der Mikrostudien reichen allerdings nur bis höchstens 2005/2006 (Kalina/Weinkopf 2008; Bosch et al. in diesem Heft). Die Frage, ob die Ungleichheit auch 2007 und 2008 weiter zunahm, kann nicht mit Mikrodaten (SOEP; IAB-Betriebspanel; Mikrozensus; EVS) beantwortet werden. Wir versuchen deswegen, Antworten aus den Daten der BA und der Volkswirtschaftlichen Gesamtrechnung (VGR) von Destatis, die bis zum ersten Quartal 2008 verfügbar sind, zu finden und somit indirekt Hinweise auf die Verteilungswirkungen der Reformen zu erhalten.

Was atypische Beschäftigung anbelangt, deutet nichts darauf hin, dass sich die Lohnschere wieder verkleinert hätte. Im jüngsten Aufschwung sind trotz der zum Teil sehr guten Konjunkturentwicklung die Löhne insgesamt nur wenig gestiegen. In den ersten elf Quartalen dieses Aufschwungs sind die Arbeitskosten je Stunde nominal nur um $2 \%$ gestiegen, im vorigen, gleich langen Zyklus war es noch ein $\mathrm{Zu}$ wachs von $7 \%$. Real - also unter Berücksichtigung des Preisanstiegs - sanken die Nettolöhne je Arbeitnehmer in diesem Konjunkturaufschwung (nach 13 Quartalen) um 3,5\% (Abbildung 1). Nach Auskunft des Bundesministeriums der Finanzen $^{4}$ hat das reale Nettoeinkommen eines Ein-Personenhaushalts in den vergangenen drei Jahren um 2,6\% abgenommen, das entsprechende Einkommen eines Vier-Personenhaushalts mit einem Alleinverdiener sank im gleichen Zeitraum um gut 3,5\%. Insofern stimmt die Wahrnehmung vieler Arbeitnehmer, nicht am Aufschwung beteiligt zu sein, sondern sogar noch zu verlieren.

Die Frage, ob die intendierte und realisierte Flexibilisierung des deutschen Arbeitsmarktes die versprochene Beschäftigungsdividende abgeworfen hat, soll anhand eines Vergleichs mit dem vorherigen Konjunkturzyklus, der nicht so stark von den Arbeitsmarktreformen beeinflusst worden war, beantwortet werden. Haben Lohnmoderation und gestiegene Lohnspreizung tatsächlich mehr Jobs geschaffen?

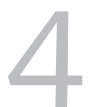

\section{Ein normaler Konjunktur- aufschwung?}

Wie der Sachverständigenrat zur Begutachtung der gesamtwirtschaftlichen Entwicklung (SVR 2007) datiert auch das IMK den Beginn des gegenwärtigen Zyklus auf das vierte Quartal 2004. Er dauerte mindestens bis zum Frühjahr 2008. Uneinheitlicher ist dagegen die Datierung des Anfangs des vorangegangenen Aufschwungs. Je nach Filterungstechnik (Horn/Rietzler 2007) liegt sein Beginn zwischen 1997 und 1999 (Zyklen, die eine Rezession einschließen, werden nicht betrachtet). Diese Einteilungen sind letztlich aber sehr ähnlich und die Ergebnisse bleiben robust, unabhängig davon, welche Einteilung man verwendet. ${ }^{5}$

\footnotetext{
4 Süddeutsche Zeitung vom 14. März 2008 sowie Bundesdrucksache 16/8346 und Bundestagsplenarprotokoll 16/141, S. 14855D-14871A vom 13. Februar 2008.

5 Siehe Horn/Logeay 2008 für eine andere Aufteilung als hier.
} 
Nimmt man eine mittlere Position ein und geht von einem Anfang im zweiten Quartal 1998 aus, dann zeigt sich auf den ersten Blick eine überraschende Parallelität zwischen dem jetzigen und dem vorangegangenen Aufschwung (Abbildung 2). Beide haben eine ähnliche Entwicklung und weisen exakt die gleiche Stärke nach elf Quartalen beim preisbereinigten Bruttoinlandsprodukt auf. Dies erlaubt einen sehr guten Vergleich der anderen Größen: Gibt es dort Abweichungen gegenüber dem vorangegangenen Zyklus, kann es nicht am Wachstum selbst liegen. Der vorherige Aufschwung war zwar nach elf Quartalen zu Ende, während der Endpunkt des jetzigen Aufschwungs noch offen ist; allerdings mehren sich die Anzeichen, dass dies in Deutschland gegenwärtig der Fall ist.

Schaut man sich die Struktur des derzeitigen Zyklus an, dann überrascht seine außenwirtschaftliche Dominanz. Er ist fast ausschließlich vom Export getragen $(+36 \%)$, während die reale Inlandsnachfrage kaum zunahm $(+5 \%)$. Im vorigen Aufschwung war dieses Muster auch vorhanden, aber weniger ausgeprägt. Es zeigt sich, dass dieses Mal selbst nach über drei Jahren außenwirtschaftlicher Stimulierung der Funke nicht auf die Binnenwirtschaft übergesprungen ist. Besonders deutlich wird dies beim realen privaten Konsum. Im vorigen Zyklus stieg er noch um insgesamt 7,5\%,- also genauso stark wie das Bruttoinlandsprodukt - jetzt stagniert er während der gesamten Aufschwungphase (insgesamt nur ein Zuwachs von weniger als $1 \%)$.

Die internationalen Preisschübe für importierte Rohstoffe, Energie und Nahrungsmittel wurden durch die Euro-Aufwertung der letzten Jahre zu einem guten Teil ausgeglichen. Allerdings hat die Erhöhung der Mehrwertsteuer um drei Prozentpunkte im Januar 2007 deutliche Preiseffekte ausgelöst (Deutsche Bundesbank 2008, S. 31ff.). Auch die administrierten Preise haben in diesem Aufschwung deutlich zugenommen (SVR 2007, Tabelle E2). Insgesamt hat der Deflator des privaten Konsums in diesem Aufschwung nach gut drei Jahren so um fast $6 \%$ zugelegt und damit zur gegenwärtigen Konsumschwäche beigetragen, während er im vorigen Aufschwung gerade einmal um knapp $2,5 \%$ zunahm

\section{Abb. 2: Kompontenten des Wachstums im Zyklenvergleich}

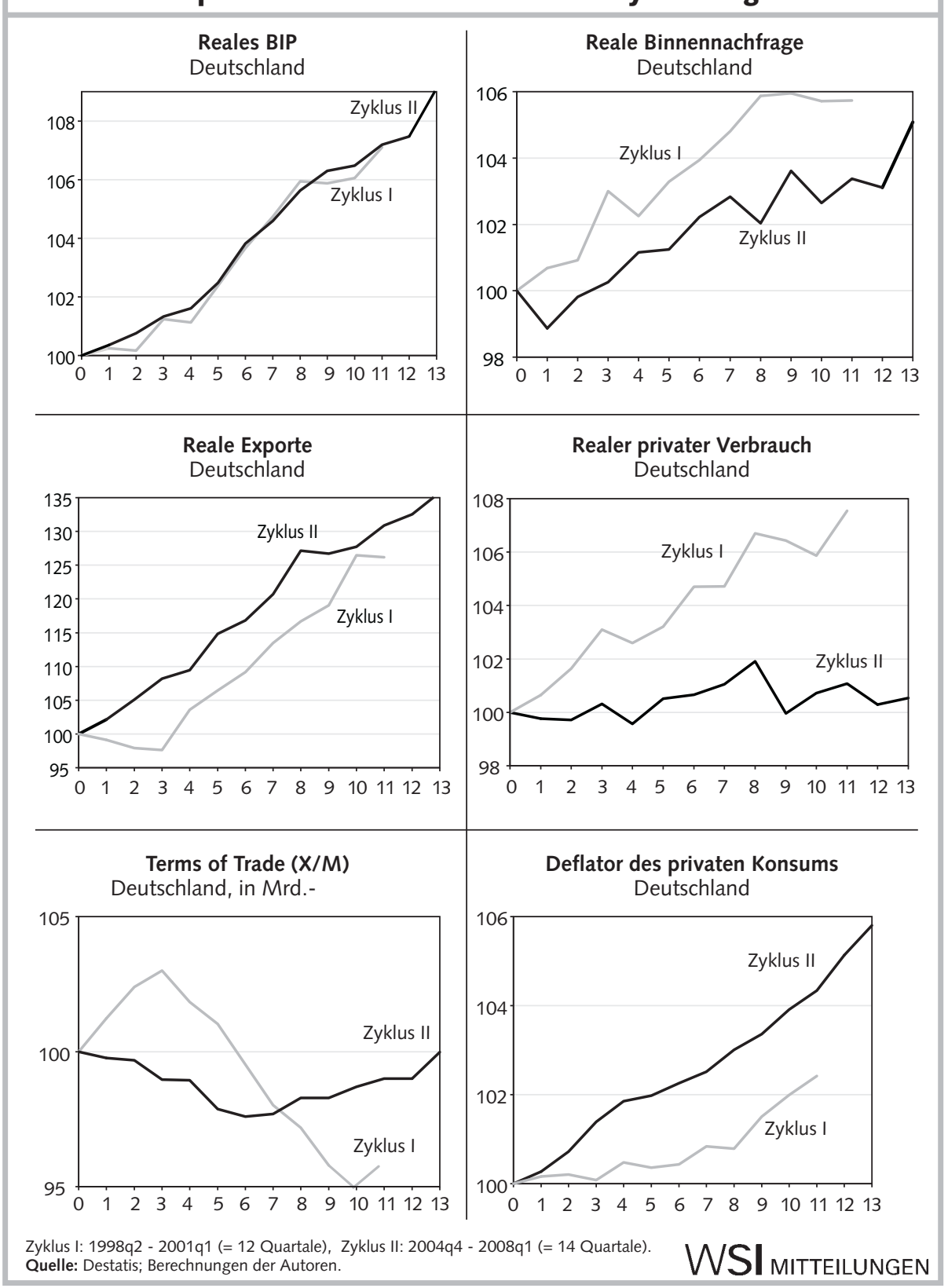

Quelle: Destatis; Berechnungen der Autoren.

\section{Nur normale Arbeitsmarkt- entwicklung}

Über einen Zeitraum von acht Quartalen hat das Arbeitsvolumen in beiden konjunkturellen Aufschwüngen ähnlich stark zugenommen, anschließend stagnierte es im vergangenen Zyklus, während es im derzeitigen Aufschwung weiter deutlich zulegte (Abbildung 3). Dennoch führte der Anstieg des Arbeitsvolumens in diesem Aufschwung bisher zu einem geringeren Arbeitsplatzaufbau (rund 0,2 Mio. Personen weniger, wie Tabelle 1 zeigt). Die Kompo- nenten der Erwerbstätigkeit lassen dabei Verschiedenes erkennen: Während die Selbstständigkeit in diesem Zyklus stärker zunahm, blieb der Anstieg bei der abhängigen Beschäftigung bislang deutlich zurück. Die Förderung der sozialversicherungspflichtigen Beschäftigung und die Förderung der Selbstständigkeit sind in diesem Zyklus stark zurückgegangen. Die ungeförderte sozialversicherungspflichtige Beschäftigung kann dennoch im elften Quartal dieses Aufschwungs einen kumuliert höheren Zuwachs ausweisen $(+816.000$ Pers.) als im vorherigen Aufschwung (+653.000 Pers.). Im vorherigen Zyklus könnte der Beschäftigungsaufbau allerdings überzeichnet gewesen sein, da ein Teil 


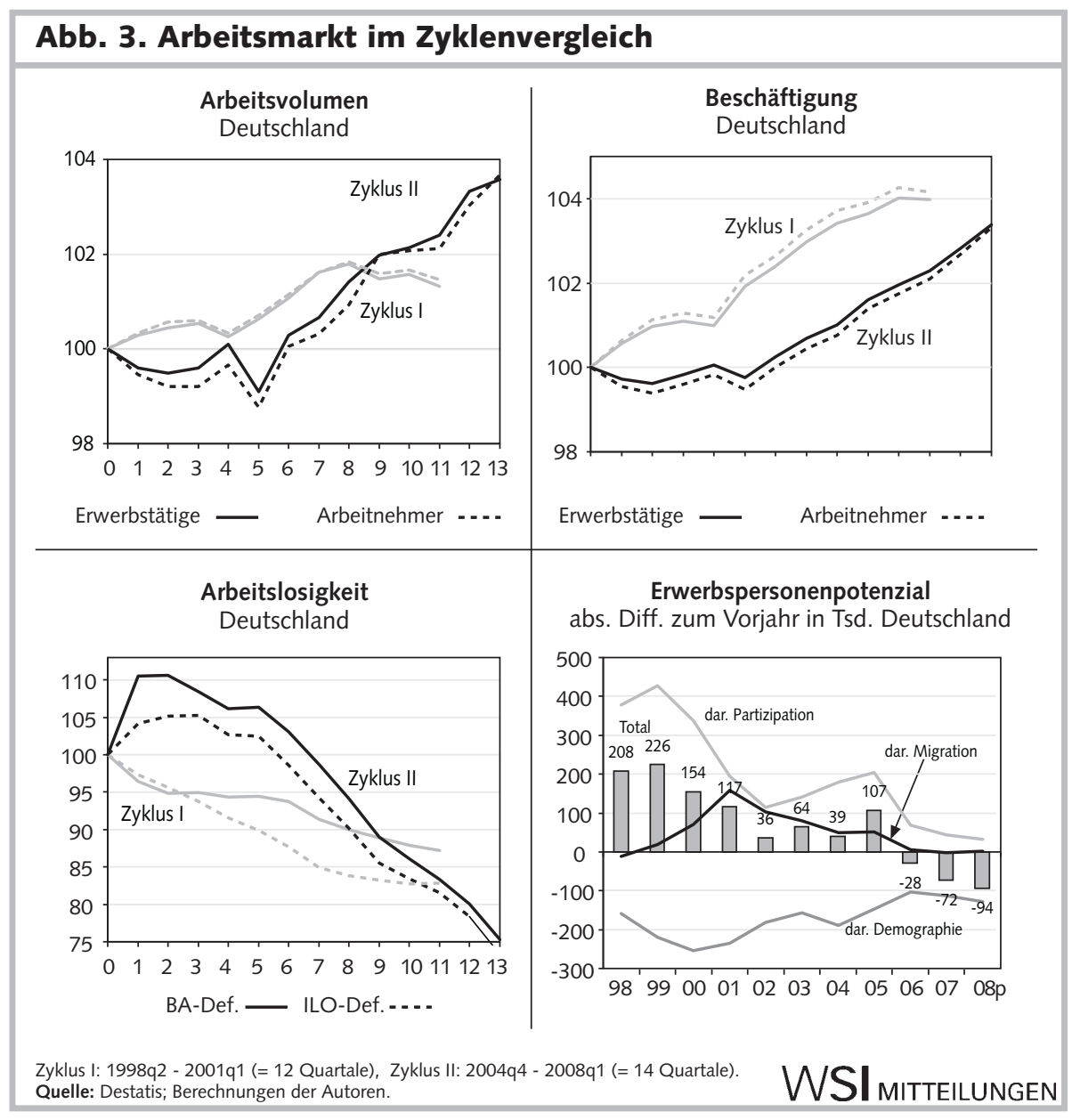

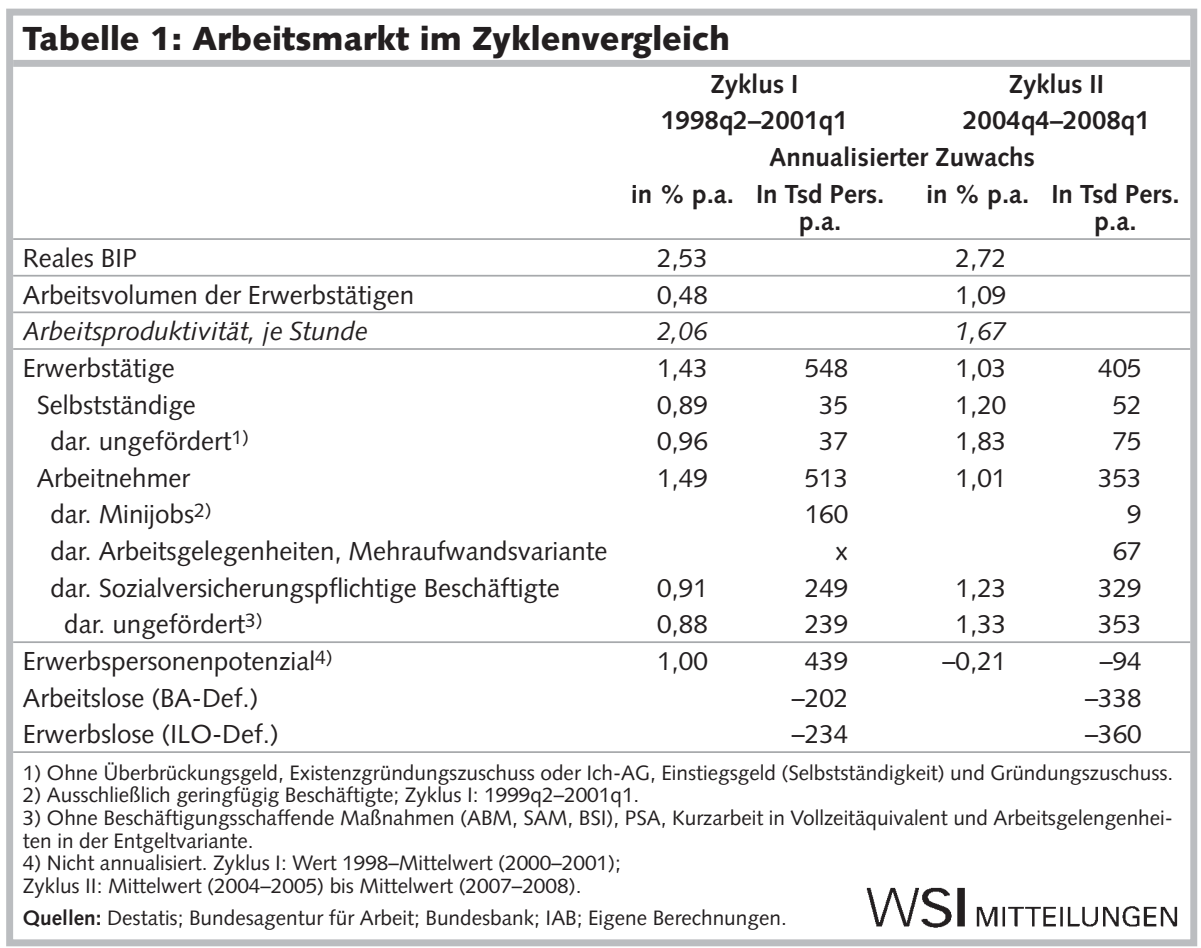

der Expansion der Minijobs (+441.000 Pers.) zulasten von ehemaligen Minijobs in Nebentätigkeit - die ähnlich wie Überstunden in der Beschäftigungsstatistik gezählt werden - stattgefunden haben dürfte. Hinjobs zu Substitutionseffekten von sozialversicherungspflichtiger Beschäftigung hin zu Minijobs geführt haben, sodass nach elf Quartalen Aufschwung Ende 2007 bei den sozialversicherungspflichtig Beschäftigten gerade das Eingangsniveau vom zweiten Quartal 1998 erreicht werden konnte. Dagegen stagnieren in diesem Aufschwung die Minijobs (+30.000 Pers.) nun auf hohem Niveau (4,9 Mio. Personen), sodass wahrscheinlich keine weitere Substitution mehr stattfindet. Da die Förderung der abhängigen Beschäftigung durch die 2005 neu eingeführten Ein-Euro-Jobs den Rückgang der traditionellen Förderung (in die sozialversicherungspflichtige Beschäftigung) vollkommen überkompensieren konnte, kann die aktive Arbeitsmarktpolitik keine Erklärung für die in diesem Zyklus geringere Dynamik bei der abhängigen Beschäftigung leisten. Im vorherigen Zyklus täuschte die starke Zunahme der Minijobs eine hohe Dynamik vor, die sich damals schon nicht in der Entwicklung des Arbeitsvolumens zeigte. Letztlich dürfte sich die Arbeitsmarktentwicklung in diesem Aufschwung normalisiert haben.

Angesichts eines im Saldo geringeren Arbeitsplatzaufbaus im Vergleich zum vorherigen Aufschwung, einer besseren Arbeitsvolumenentwicklung und einer ähnlichen Entwicklung bei den sozialversicherungspflichtigen Jobs während der ersten elf Quartale zeigen sich insgesamt keine positiven Wirkungen der Arbeitsmarktreformen. Diese werden zwar immer wieder behauptet, doch der Beweis wird nicht erbracht (siehe die Beiträge verschiedener Autoren in Wirtschaftsdienst 3/2008). Der Unterschied besteht im Wesentlichen darin, dass der derzeitige Aufschwung dank der besseren weltwirtschaftlichen Entwicklung länger andauert. Dass die Arbeitslosigkeit in diesem Aufschwung vergleichsweise stärker abgesunken ist (Abbildung 3; Tabelle 1), hat noch einen weiteren Grund: Das Arbeitsangebot geht derzeit zurück, während es im vorherigen Aufschwung noch relativ stark zunahm. Dies zeigen die untergliederten Abgänge aus der Arbeitslosigkeit. ${ }^{6}$ Neben der guten Konjunktur ist also ein nicht zu vernachlässigender Arbeitsangebotseffekt am Werk. Die Zahl der Arbeitslosen ist auch noch stark von den

\footnotetext{
6 Die Abgänge aus der Arbeitslosigkeit insgesamt waren im Durchschnitt 609.019 (1999-2000) bzw. 665.517 (2005-2007) Personen pro Monat. Nimmt man aber nur die Abgänge aus der Arbeitslosigkeit in die Erwerbstätigkeit, reduzieren sich die Unterschiede deutlich auf 270.613 bzw. 273.046 (Bundesagentur für Arbeit, diverse Monatsberichte)
} 
statistischen Effekten der Einführung von Hartz IV geprägt; nach der außerordentlichen Zunahme im Januar 2005 werden bis heute die Statistiken durch die ,intensivere Betreuung von Arbeitslosen sowie die systematische Überprüfung des Arbeitslosenstatus" bereinigt (Monatsberichte der BA; IMK 2005, S. 16f. für eine Abschätzung; Abbildung 3; Tabelle 1). Dass der Anteil der älteren Arbeitslosen ganz leicht zurückging, dürfte in erster Linie auf die Reduzierung von Möglichkeiten zur Frühverrentung zurückzuführen sein. Angesichts des gestiegenen Anteils von älteren Arbeitnehmern unter den Beschäftigten insgesamt hätte der Anteil der Älteren unter den Arbeitslosen eigentlich stärker abnehmen müssen (IMK 2007, S. 16ff.). Dies weist auf die - trotz Reformen - eher steigenden Vermittlungsschwierigkeiten bei den über 55-Jährigen hin.

\section{Ausufernde Gewinnentwicklung}

Im Gegensatz zu den geringen Lohnsteigerungen sind die Gewinne der Unternehmen (und Vermögenseinkommen) geradezu explodiert. Sie stiegen in den elf Quartalen dieses Aufschwungs um $25 \%$. Im vorigen Aufschwung war es nur ein Zuwachs von $5 \%$. Letztlich konnte der Aufschwung dieses Mal von den Unternehmen für eine massive Umverteilung zu ihren Gunsten genutzt werden. Und das, obwohl der Staat mit seinem Defizitabbau reduzierend auf Gewinne und Nachfrage gewirkt hat. Letztlich waren die Unternehmen bei guter internationaler und nationaler Konjunktur nicht gezwungen, die geringen Arbeitskostensteigerungen vollständig in den Preisen weiterzugeben und diese - bei Einrechnung der Produktivitätsfortschritte - entsprechend zu senken. Stattdessen konnten sie ihre Gewinnmargen erhöhen. In Unternehmensberichten wird daher von steigenden Kapital- und Umsatzrenditen gesprochen.

Bei dieser Entwicklung von Löhnen und Gewinnen ging die Lohnquote weiter deutlich zurück. Schwankte sie im letzten Aufschwung noch um einen Anteil von $71 \%$ am Volkseinkommen, sackte sie in diesem Aufschwung von $68 \%$ auf rund $64 \%$ ab. Im gleichen Ausmaß wie die Lohnquote gesunken ist, ist die Gewinnquote in diesem Aufschwung gestiegen.

Abb. 4: Verteilung des verfügbaren Einkommens im Zyklenvergleich

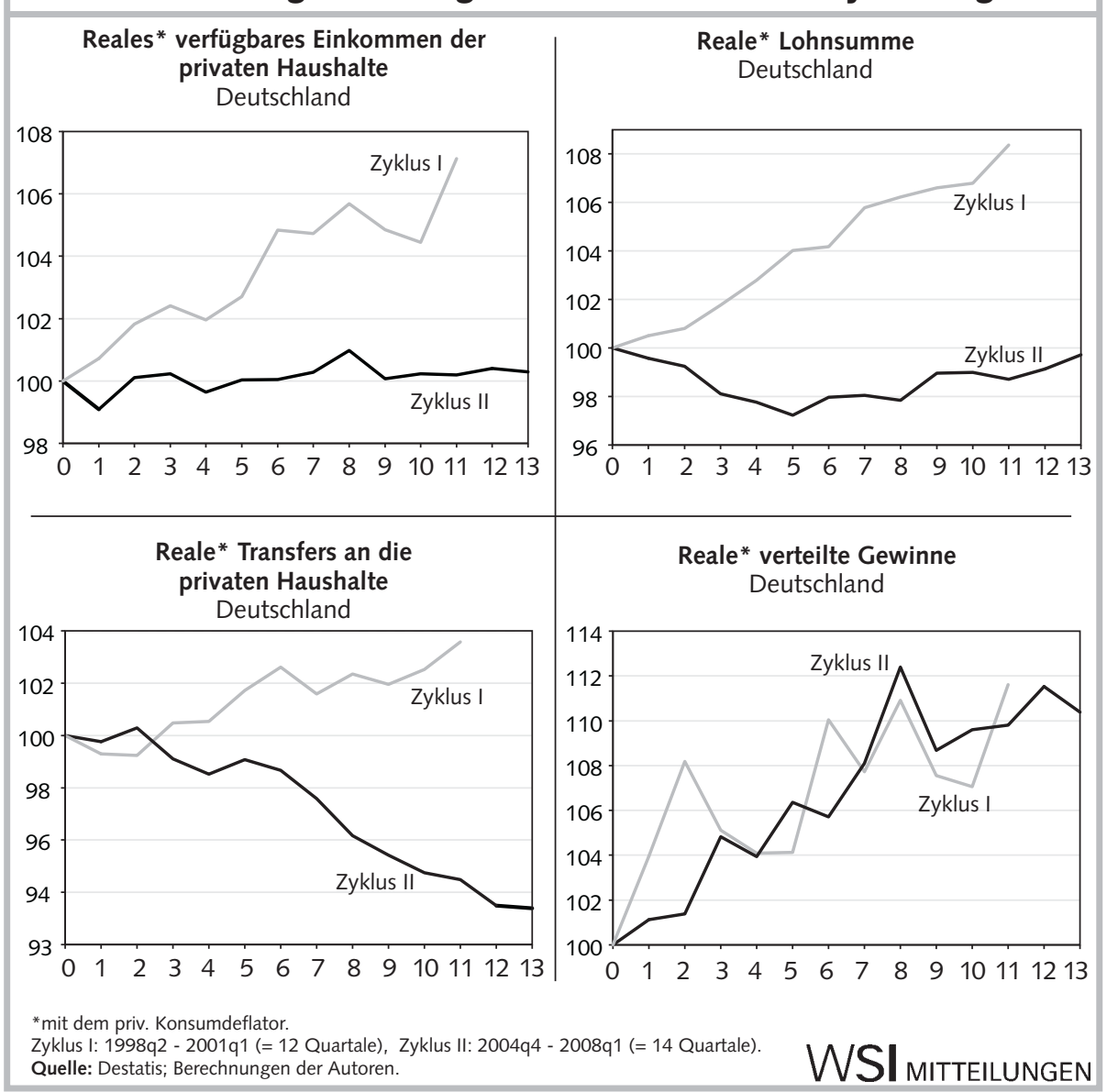

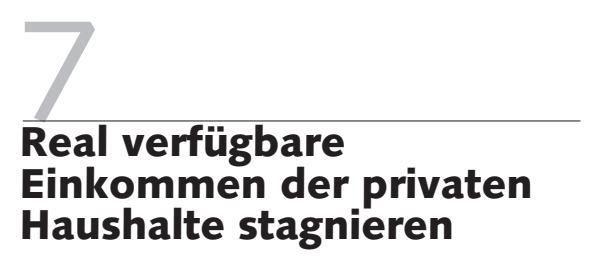

Niedrige Lohnzuwächse und hohe Gewinnsteigerungen bestimmen allein noch nicht die finanzielle Situation der privaten Haushalte. Diese hängt darüber hinaus stark von den Umverteilungsmaßnahmen des Staates über Steuern und Beiträge und insbesondere von den Transferzahlungen an die privaten Haushalte sowie von der Entwicklung der Beschäftigung ab. Auch beeinflusst die Preisentwicklung die reale Einkommenssituation.

Im Folgenden werden daher die drei Komponenten des verfügbaren Einkommens der privaten Haushalte näher betrachtet: die Nettolohn- und Gehaltssumme, die Transfers und die entnommenen Gewinne und Vermögenseinkommen. Alle drei Komponenten werden mit dem Deflator des privaten Konsums bereinigt, um so ihre reale Entwicklung darstellen zu können. Anschließend werden sie mit ihrer je- weiligen Entwicklung im vorherigen Aufschwung verglichen (Abbildung 4).

Die Ergebnisse zeigen, dass trotz eines Beschäftigungsanstiegs um 3,3 \% in diesem Aufschwung alle Beschäftigten zusammen nur über ein um $0,3 \%$ rückläufiges reales Nettolohneinkommen verfügten. Im vorherigen Aufschwung hatten sie dagegen noch einen Zuwachs von über $8 \%$ für sich verbuchen können. In dieser Entwicklung zeigen sich neben den Wirkungen der niedrigen Lohnzuwächse auch die Effekte der Konsolidierungsstrategie des Staates. Insbesondere die Anhebung der Mehrwertsteuer um drei Prozentpunkte hat die reale Einkommensentwicklung deutlich geschwächt. Noch gravierender waren allerdings die Auswirkungen bei den Transferempfängern. Die realen Transfers an die privaten Haushalte sind in diesem Aufschwung um über $6 \%$ zurückgegangen. Im vorigen Aufschwung waren sie noch um rund $4 \%$ gestiegen. Dahinter verbergen sich die Nullrunden bei den nominalen Renten, stagnierende nominale Leistungen bei Kindergeld, Bafög und anderen staatlichen Leistungen. Einen Anteil hat aber auch die niedrigere Zahl an Arbeitslosen. Letztlich wurde das 
Ziel der Reduzierung staatlicher Transferzahlungen von der Politik erreicht.

Außerordentlich gut verlief dagegen die Entwicklung der realen entnommenen Gewinne und Vermögenseinkommen (über $10 \%$ Zuwachs). Dabei muss berücksichtigt werden, dass nicht alle Gewinne ausgeschüttet werden, ein Teil verbleibt im Unternehmen. Und dennoch konnten sich Unternehmer, Selbstständige, Aktienbesitzer und andere Kapitaleigner über einen stetig steigenden Strom an Einnahmen freuen, der auch von der Preisentwicklung nicht aufgezehrt wurde. Dabei darf nicht übersehen werden, dass sich auch in diesem Kreis etliche Ein-Personen-Unternehmen und andere Selbstständige befinden, die nicht von dieser Entwicklung profitierten. Umso höher fielen die Einkommenszuwächse der anderen aus.

Insgesamt ist das preisbereinigte verfügbare Einkommen der privaten Haushalte in diesem Aufschwung nicht gestiegen. Das dürfte eine neue Qualität eines Konjunkturaufschwungs darstellen, wie es sie früher vermutlich noch nie gegeben hat: Die wirtschaftliche Leistung wächst deutlich, doch bei der Mehrzahl der privaten Haushalte kommt davon überhaupt nichts an. Im vorigen Aufschwung war das real verfügbare Einkommen der privaten Haushalte immerhin um $7 \%$ gestiegen und lag damit genauso hoch wie das Wirtschaftswachstum.

\section{Fazit}

Steigende Preise für Nahrungsmittel und Energie werden auch in diesem Jahr die Realeinkommensentwicklung dämpfen. Das aber heißt: Der von vielen als Belohnung für die Lohnzurückhaltung versprochene zusätzliche Beschäftigungsanstieg hat sich nicht eingestellt und wird sich nicht einstellen. Bei gleich starkem Wachstum wie im vorherigen Aufschwung in den ersten elf Quartalen und trotz eines insgesamt länger andauernden Aufschwungs hat die Zahl der abhängig Beschäftigten insgesamt dieses Mal etwas weniger, die der sozialversicherungspflichtigen Beschäftigten dagegen etwas stärker zugenommen als im vorherigen Aufschwung. Die versprochene Beschäftigungsdividende für Lohnmoderation und Arbeitsmarktflexibilisierung fiel damit aus, der - erzwungene - Verzicht auf angemessene, an Inflationsziel und Produktivitätssteigerung orientierte Lohnsteigerungen hat sich nicht gelohnt. Dafür stiegen die Dividenden und Kursgewinne der Aktienbesitzer umso mehr. Die Gewinne der Unternehmen sind geradezu explodiert. Bei nachlassender Weltkonjunktur und schwacher Binnennachfrage werden diese Gewinne aber nicht automatisch investiert. Und da die Sparquote von Ge- winneinkommensbeziehern überdurchschnittlich hoch ist, leidet der private Konsum nicht nur unter den gestiegenen Sparanstrengungen der Arbeitnehmer, sondern auch unter der Umverteilung von den Löhnen hin zu den Gewinnen (Deutsche Bundesbank 2007b). Da die Rentenhöhe direkt von den vorangegangenen Lohnsteigerungen abhängt, leiden auch die Rentner und andere Transfereinkommensbezieher unter dieser Umverteilung. Nach jahrelangen realen Rentenkürzungen wird sich dies in diesem Jahr nicht mehr ändern. Viele andere Transferzahlungen, wie z. B. Arbeitslosengeld, Kindergeld und Bafög, wurden ebenfalls jahrelang nicht angehoben und damit durch die Inflation zunehmend entwertet.

Die Fakten und Analysen dieses Beitrags wie auch die anderer Studien sind eindeutig: Es ist nicht nur ein mehrheitliches Gefühl der Bevölkerung, dass sie vom Aufschwung nicht profitiert. Es ist Realität. Trotz eines gut dreijährigen Konjunkturaufschwungs ist die reale Einkommenssituation vieler Haushalte heute schlechter als zuvor. Und dank der Reformen müssen sie heute auch mehr privat für das Alter und andere Lebensrisiken vorsorgen. Wer also hat vom Aufschwung profitiert? Nicht die Masse der Arbeitnehmer und nicht die Transfereinkommensbezieher. Wohl aber die Unternehmensvorstände, die Vermögensbesitzer und viele Selbstständige.

\section{LITERATUR}

Bassanini, A./Duval, R. (2006): Employment Patterns in OECD Countries: Reassessing the Role of Policies and Institutions, Social, employment and migration, OECD-working-papers 35, download: http://www.oecd. org/document/21/0,3343,fr_2649_33927_35427733_1_1_1_1,00.html Bispinck, R. (2003): Das deutsche Tarifsystem in Zeiten der Krise - Streit um Flächentarif, Differenzierung und Mindeststandards, in: WSI-Mitteilungen 7, S. 395-404

Bispinck, R./Schulten, T. (2005): Deutschland vor dem tarifpolitischen Systemwechsel?, in: WSI-Mitteilungen 8, S. 466-472

Bispinck, R./WSI-Tarifarchiv (1999): Das Märchen vom starren Flächentarifvertrag - Eine Analyse von tariflichen Öffnungsklauseln aus über 100 Tarifbereichen, Elemente qualitativer Tarifpolitik 37, Düsseldorf

Bispinck, R./WSI-Tarifarchiv (2005): Immer flexibler - und immer länger? Tarifliche Regelungen zur Arbeitszeit und ihrer Gestaltung. Eine Analyse von 24 Tarifbereichen, Elemente qualitativer Tarifpolitik 57, Düsseldorf
Bispinck, R./WSI-Tarifarchiv (2007): Unterste Tarifvergütungen 2007 Ausgewählte Tarifbereiche mit unterster Tarifgruppe und Laufzeitende der Vergütungstarifverträge, Elemente qualitativer Tarifpolitik 63, Düsseldorf

Bosch, G./Kalina, T. (2007): Niedriglöhne in Deutschland - Zahlen, Fakten, Ursachen, in: Bosch G./Weinkopf C. (Hrsg.): Arbeiten für wenig Geld - Niedriglohnbeschäftigung in Deutschland, Frankfurt/Main, S. 20-105 Bothfeld, S. (2007): Labour Market Institutions in Germany: Current Status and Ongoing Reforms, WSI-Discussion Paper 152

Brandt, T./Drews, K./Schulten, T. (2007): Liberalisierung des deutschen Postsektors - Auswirkungen auf Beschäftigung und Tarifpolitik, in: WSIMitteilungen 5, S. 266-273

Brenke, K. (2007): Zunehmende Lohnspreizung in Deutschland, DIW Wochenbericht 6, S. 73-79 
Bundesagentur für Arbeit (BA) (2004): Mini- und Midijobs in Deutschland, Sonderbericht 12, Nürnberg

Destatis (2006): Datenreport 2006, Zahlen und Fakten über die BRD, Wiesbaden, Kap. 18

Deutsche Bundesbank (2005a): Monatsbericht 2, Frankfurt/Main Deutsche Bundesbank (2005b): Monatsbericht 7, Frankfurt/Main Deutsche Bundesbank (2007a): Monatsbericht 9, Frankfurt/Main Deutsche Bundesbank (2007b): Monatsbericht 10, Frankfurt/Main Deutsche Bundesbank (2008): Monatsbericht 4, Frankfurt/Main Ebbinghaus B./Eichhorst W. (2006): Employment Regulation and Labor Market Policy in Germany 1991-2005, IZA-Discussion Paper 2505 Europäische Kommission (EU) (2004): Employment in Europe 2004 Recent Trends and Prospects. European Commission, Directorate-General for Employment and Social Affairs, Bruxelles

Europäische Kommission (EU) (2007): Raising Germany's growth potential. European Economy - Occasional Papers 28, Brüssel

Fertig, M./Kluve, J./Scheuer, M. (2005): Was hat die Reform der Minijobs bewirkt? Erfahrungen nach einem Jahr, RWI-Schriften 77, Berlin Gernandt, J./Pfeiffer, F. (2007): Zunehmende Ungleichheit der Arbeitsverdienste, in: ZEW Wachstums- und Konjunkturanalysen, September,

S.10-11

Grabka, M. M./Frick, J. (2008): Schrumpfende Mittelschicht - Anzeichen einer dauerhaften Polarisierung der verfügbaren Einkommen?, DIW Wochenbericht 10

Horn, G./Logeay, C. (2008): Gibt es eine Reformdividende? Eine vorläufige Bilanz der Arbeitsmarktreformen, in: Wirtschaftsdienst 3, Zeitgespräch, S. $160-165$

Horn, G./Logeay, C. /Zwiener, R. (2008): Wer profitierte vom Aufschwung, IMK-Report 27

Horn, G./Logeay, C./Stephan, S./Zwiener, R. (2007): Preiswerte Arbeit in Deutschland - Auswertung der aktuellen Eurostat Arbeitskostenstatistik, IMK-Report 22
Horn, G./Rietzler, K. (2007): Forcierte Angebotspolitik löst keinen zusätzlichen Investitionsschub aus. Ein Zyklenvergleich, IMK-Report 24 IMK (2005): Wirtschaftliche Entwicklung 2006: Vor schwierigen Weichenstellungen, IMK-Report 3

IMK (2007): Der Abschwung kommt, IMK Report 25

Kalina, T./Weinkopf, C. (2008): Weitere Zunahme der Niedriglohnbeschäftigung: 2006 bereits rund 6,5 Millionen Beschäftigte betroffen, IAQ-Report 1

Keller, B./Seifert, H. (2007): Atypische Beschäftigungsverhältnisse: Flexibilität, soziale Sicherheit und Prekarität, in: Keller, B./Seifert, H. (Hrsg.): Atypische Beschäftigung - Flexibilisierung und soziale Risiken, Berlin, S. $11-25$

Nienhüser, W./Matiaske, W. (2003): Der "Gleichheitsgrundsatz" bei Leiharbeit - Entlohnung und Arbeitsbedingungen von Leiharbeitern im europäischen Vergleich, in: WSI-Mitteilungen 8, S. 466-473

OECD (2008): OECD Economic Surveys - Germany 7

Promberger, M. (2006): Leiharbeit - Flexibilität und Prekarität in der betrieblichen Praxis, in: WSI-Mitteilungen 5, S. 263-268

Promberger, M. (2008): Leiharbeit: Zwischenbilanz und offene Fragen, in: WSI-Mitteilungen 4, S. 223-225

Rhein, T./Stamm, S. (2006): Niedriglohnbeschäftigung in Deutschland deskriptive Befunde zur Entwicklung seit 1980 und Verteilung auf Berufe und Wirtschaftszweige, IAB-Forschungsbericht 12

Rudolph, H. (2003): Mini- und Midi-Jobs: Geringfügige Beschäftigung im neuen Outfit, IAB-Kurzbericht 6

Sachverständigenrat zur Begutachtung der gesamtwirtschaftlichen Entwicklung (SVR) (2004): Jahresgutachten 2004/2005, Erfolge im Ausland - Herausforderungen im Inland, Ziffern 235-267, Wiesbaden Sachverständigenrat zur Begutachtung der gesamtwirtschaftlichen Entwicklung (SVR) (2007): Jahresgutachten 2007/2008, Das Erreichte nicht verspielen, Ziffern 481-492, Wiesbaden 